\title{
Design of Ultra Fast RCE Photodetectors for Optical Communications systems
}

\author{
Joseph A. Jervase and Hadj Bourdoucen
}

Department of Information Engineering, College of Engineering, Sultan Qaboos University, P.O. Box 33, Al Khod 123, Muscat, Sultanate of Oman. Email: Jervase@squ.edu.om.
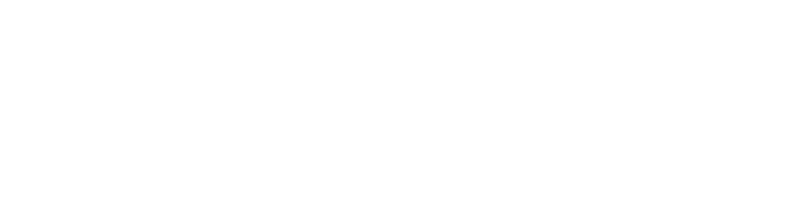

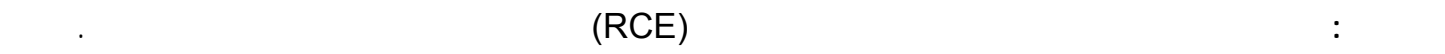

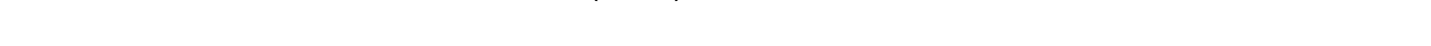

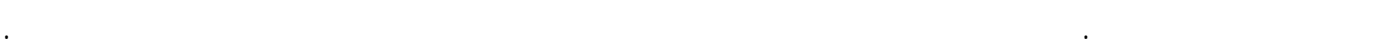

\begin{abstract}
Two key parameters for RCE photodetectors that govern their suitability for ultrafast optical communication systems are considered. These are the quantum efficiency and the bandwidth efficiency product. A closed analytical form has been derived for quantum efficiency, which incorporates the structural parameters of the photodetector. Based on the simulation results, an optimization and design procedure for these photodetectors has been developed.
\end{abstract}

KEYWORDS: Cavity Resonators, Photodetectors, Photodiodes, Quantum Efficiency and ResonantCavity-Enhanced Photodetectors.

\section{Introduction}

$\mathbf{O}$ ptical photodetectors are the key components in optical communication systems. Their quantum efficiency and optical bandwidth characteristics are of major importance in the design of fiber optic based data communication systems. A number of structures and design optimization techniques have been proposed in the literature to achieve high values for these two parameters (Kishino, 1991), (Unlu, 1992, 1995, 1998), (Tan, 1995), (Murtaza 1996), (Tung, 1997), (Jervase, 1998, 2000). One of the promising types is the Resonant Cavity Enhanced (RCE) photodetector. In the RCE, the active region is placed inside a Fabry-Perot cavity between two mirrors made of quarter-wave stacks (QWS) to allow the signal light to have more than one absorbing path inside the active region (Kishino, 1991), (Unlu, 1992, 1995, 1998), (Tan, 1995), (Murtaza, 1996), (Tung, 1997), (Jervase, 1998, 2000), (Onat, 1998), (Ozbay, 1997). The resulting structure will need only a very thin absorbing intrinsic region to achieve high quantum efficiency as well as a high speed of response. Hence, RCE photodiodes with quantum efficiencies close to unity can be designed.

In this work, the quantum efficiency is formulated in a closed analytical form. This includes the structural parameters of the photodetector and takes into consideration the wavelength dependence of the end mirrors reflectivities and the absorption coefficient of the intrinsic region. Using this formulation, an optimization and design procedure for RCE photodetectors has been developed and design charts have been generated with the quantum efficiency, quality factor and frequency bandwidth as input design parameters. 


\section{Analysis}

Figure 1 shows the integrated device of our investigation, which is based on the system of heterojunctions $\mathrm{In}_{0.53} \mathrm{Ga}_{0.47} \mathrm{As} / \mathrm{InP}$. The speed response for this type of PIN structure is mainly limited by the transit time and the diffusion time of photogenerated carriers in the space charge region and the neutral regions. It is also limited by the charging and discharging time for traps at the heterojunction interfaces and by the inherent and parasitic capacitances of the structure. The last two effects can be minimized by incorporating non-absorbing lateral layers having graded heterojunctions with the active region and to have the heterojunctions and the active region depleted of any trapped charges that might be stored. In addition, the introduction of super lattice bandgap grading layers can eliminate the interface hole trapping process and consequently decrease the capacitive effect on the generated carriers. To further improve the frequency bandwidth requirements, the active region should be shortened enough to minimize the transit time of the photogenerated carriers crossing it. To reduce the device dark current, several types of junction architectures have been proposed based on the enhancement of the barrier heights at the interfaces of the InGaAs active layer and the adjacent layers to decrease tunneling currents of minority carriers. PIN photodetectors based on InGaAs/InP have been analyzed by many researchers e.g. (Bottcher, 1992) and the references therein). In fact, the typical PIN photodiode configuration (Dentan, 1990) has been integrated in our proposed configuration for its simplicity and ease of fabrication as well as its realistic mathematical description.

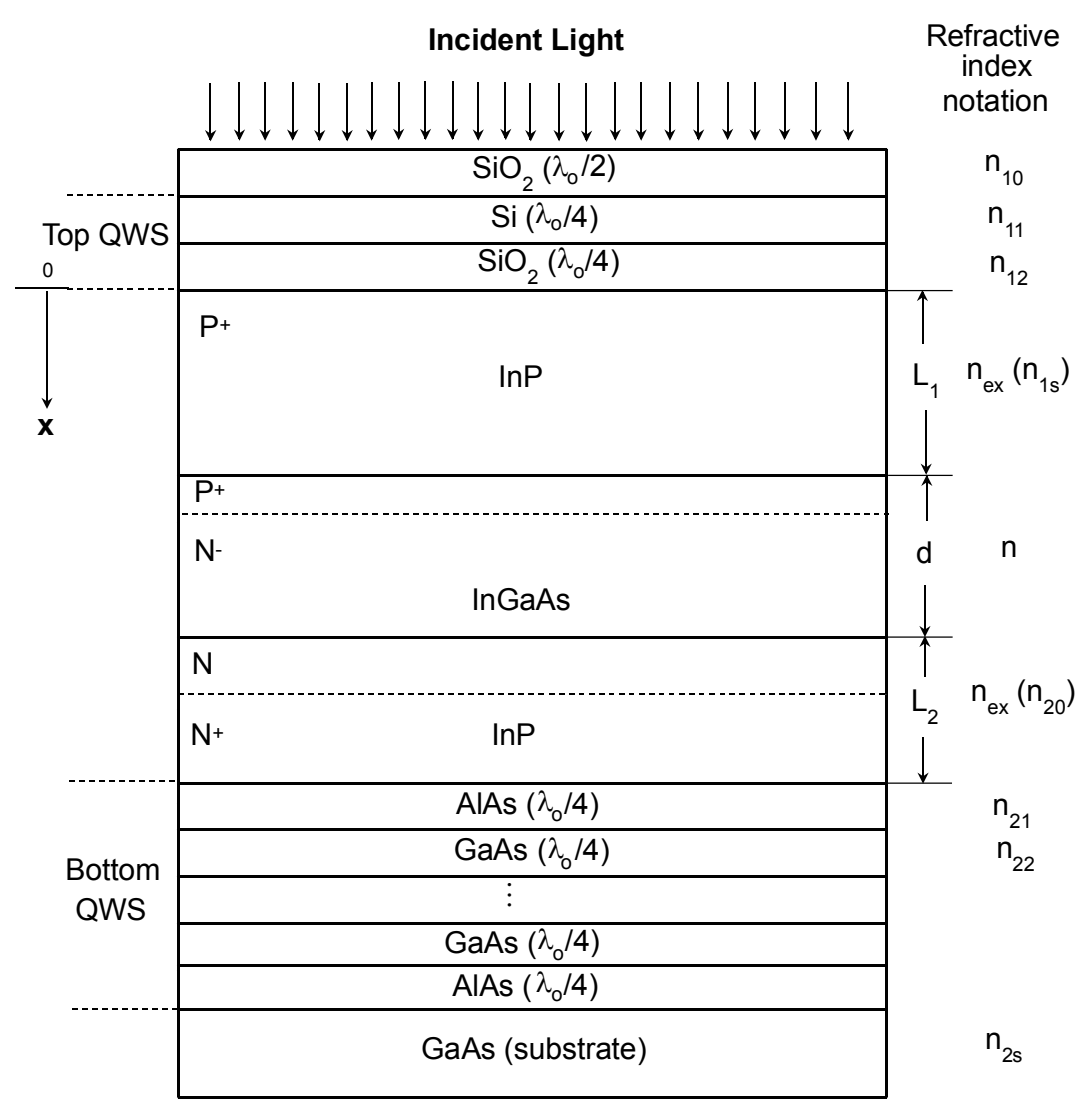

Figure 1. The RCE photodetector model.

The expression for quantum efficiency for the RCE photodetector shown in Figure 1 will be derived following a similar approach to that used in (Kishino, 1991) and (Jervase, 1998). This technique is based on finding the reflectivities of the top and bottom mirrors separately 


\section{DESIGN OF ULTR FAST RCE PHOTODETECTORS}

and then using their values to determine the fields inside the cavity by considering the whole structure of the photodetector i.e. mirrors and cavity together.

With reference to the device model shown in Figure 1 for the RCE photodetector, the quantum efficiency due to the power absorbed in the $i$-region may be expressed as follows,

$$
\eta=\frac{\left(1-R_{1}\right)\left(1+R_{2} e^{-\alpha d}\right)\left(1-e^{-\alpha d}\right)}{1+R_{1} R_{2} e^{-2 \alpha d} \cos \phi}
$$

where,

$$
\begin{gathered}
\phi=\left\{2\left[\beta_{e x}\left(L_{1}+L_{2}\right)+\beta d\right]+\psi_{1}+\psi_{2}\right\} \\
\beta_{e x}=\frac{2 \pi n_{e x}}{\lambda} \\
\beta=\frac{2 \pi n}{\lambda}
\end{gathered}
$$

In the derivation of Eq. 1, the p- and n-regions are assumed to be transparent at the design resonant wavelength $\lambda_{0}=1.3 \mu \mathrm{m}$. Thus $\alpha_{\mathrm{ex}}=0$ for the material adopted in the model (InP) at this wavelength.

It is apparent from Eq. 1 that the external quantum efficiency depends on the design of the top and bottom QWS's $\left(\mathrm{R}_{1}, \psi_{1}, \mathrm{R}_{2}, \psi_{2}\right)$, the choice of the materials for the cavity $\left(\alpha, \mathrm{n}, \mathrm{n}_{\mathrm{ex}}\right)$ and its physical dimensions $\left(\mathrm{d}, \mathrm{L}_{1}, \mathrm{~L}_{2}\right)$ as well as the operating wavelength $(\lambda)$. Within the range of interest in this analysis $(1.2 \mu \mathrm{m}<\lambda<1.5 \mu \mathrm{m})$, there is no much variation in refractive indices of the different layers of the photodetector. This does not apply, however, to the $i$ region attenuation constant $\alpha$. It has been shown in the literature that the absorption coefficient $\alpha$ of $\operatorname{In}_{0.53} \mathrm{Ga}_{0.47} \mathrm{As}$ is a function of wavelength and doping (Humphreys, 1985). Using the experimental results in (Humphreys, 1985), a nonlinear curve fitting technique has been used to obtain analytical expressions for $\alpha$.

With reference to Eq. 1, the maximum quantum efficiency occurs when $2\left[\beta_{e x}(\lambda)\left(L_{1}+L_{2}\right)+\beta(\lambda) d\right]+\psi_{1}(\lambda)+\psi_{2}(\lambda)=2 \pi$, or multiples thereof. It then follows that (Kishino, 1991), (Jervase, 1998),

$$
\eta_{\text {peak }}=\frac{\left(1-R_{1}\right)\left(1+R_{2} e^{-\alpha d}\right)\left(1-e^{-\alpha d}\right)}{1+R_{1} R_{2} e^{-2 \alpha d}}
$$

This expression is used in a constrained optimization procedure for the design of RCE photodetectors with maximum quantum efficiency. Equation 5 serves as a check on the optimized values $\left(\mathrm{L}_{1}, \mathrm{~L}_{2}, \mathrm{~d}\right)$ in whether they yield the maximum quantum efficiency.

The design procedure developed is summarized below:

Step 1: Specify the resonant wavelength $\lambda_{0}$, the quantum efficiency $\eta$, the quality factor $Q$ and the frequency bandwidth BW.

Step 2: Select the materials for the cavity and the quarter-wave-stacks (QWS's).

Step 3: Design the QWS's with the following guidelines:

Choose the number of layers $\mathrm{N}_{2}$ to achieve a reflectivity $\mathrm{R}_{2}$ at $\lambda_{0}$ close to unity for the bottom QWS.

Choose the number of layers $\mathrm{N}_{1}$ to achieve a reflectivity $0.6<\mathrm{R}_{1}<0.9$ at $\lambda_{0}$ for the top QW

Step 4: With the values of $\mathrm{R}_{1}$ and $\mathrm{R}_{2}$ now known, invoke a search and optimization program to determine $\mathrm{d}, \mathrm{L}_{1}$ and $\mathrm{L}_{2}$.

Step 5: Generate values for $\eta$ for a range of wavelengths centered at $\lambda_{0}$.

Step 6: For each set of $\mathrm{d}, \mathrm{L}_{1}$ and $\mathrm{L}_{2}$, deduce FWHM and compute the quality factor and the frequency bandwidth.

Step 7: Select the values of $\mathrm{d}, \mathrm{L}_{1}$ and $\mathrm{L}_{2}$ that satisfy the design criteria on $\mathrm{Q}, \eta$ and $\mathrm{BW}$. 


\section{Results}

The resonant wavelength used in this work is $\lambda_{0}=1.3 \mu \mathrm{m}$. The refractive indices of the various layers of the model adopted in the analysis (Figure1), are $\mathrm{SiO}_{2}(1.46)$, $\mathrm{Si} \mathrm{(3.46),} \mathrm{InP}$ (3.20), InAlGaAs (3.50) and InGaAs (3.73-j0.12).

Following the design procedure outlined earlier, 42 pairs of layers were needed to achieve a reflectivity of 0.998 at $\lambda_{0}$. For the top QWS, only one pair of layers is needed. This yields a reflectivity of 0.8 . The resonance condition to achieve maximum quantum efficiency $\left.2 \mid \beta_{\text {ex }}(\lambda)\left(L_{1}+L_{2}\right)+\beta(\lambda) d\right]+\psi_{1}(\lambda)+\psi_{2}(\lambda)=2 \pi$ may thus be expressed for the adopted structure as

$$
n_{e x}\left(L_{1}+L_{2}\right)+n d=M\left(\lambda_{0} / 2\right)
$$

where, $\mathrm{M}=1,2,3, \ldots$

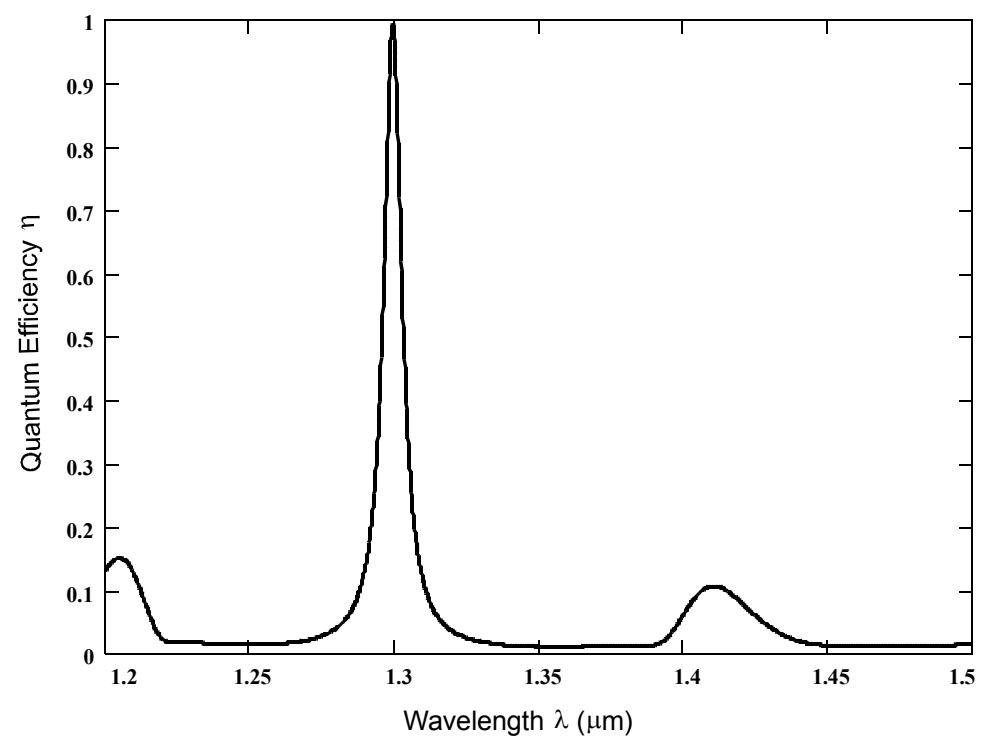

Figure 2. Variation of external quantum efficiency $\eta$ with wavelenghth $\lambda$.

Figure 2 shows the variation of external quantum efficiency with wavelength using a set of generated values $\left(d=0.1 \mu \mathrm{m}, L_{1}=L_{2}=1.465 \mu \mathrm{m}\right)$ in Eq.1. This Figure shows a dominant peak centered at $\lambda_{0}=1.3 \mu \mathrm{m}$, the design wavelength. This result is physically more realistic since the proposed structure is acting as a resonant cavity filtering out all wavelengths other than $\lambda_{0}$.

The theoretical result is also in very good agreement with recently published experimental data on high-speed RCE Schottky photodiodes (Jervase, 2000), (Unlu, 1998), (Onat, 1998) and high-speed Si-based RCE photodetectors (Ozbay, 1997). With reference to Figure 2, the use of the free spectral range (FSR) to the wavelength full width at half maximum (FWHM) as a measure of wavelength selectivity of detection (Kishino, 1991) is no longer valid. The conventional quality factor definition used for filters design defined as the ratio of $\lambda_{0}$ to the FWHM is more appropriate in this case as a measure of selectivity (Jervase, 2000).

It has been noticed that ignoring the variation of the attenuation constant $\alpha$ with $\lambda$ results in a higher off-resonance peak for $\lambda>\lambda_{0}$ and lower off-resonance peak for $\lambda_{0}$. This is due to the fact that $\alpha$ monotonically decreases with $\lambda$.

Following a design and optimization procedure, all the sets of values obtained previously for $\mathrm{d}, \mathrm{L}_{1}$ and $\mathrm{L}_{2}$ were used to obtain the quality factor $\mathrm{Q}$ corresponding to every spectrum of quantum efficiency $\eta$. The objective is to obtain design charts relating the quality factor $Q$ to the maximum achievable quantum efficiency $\eta_{\max }$ for a given structure dimensions. The 
curves of Figure 3 show the interdependence of the quality factor $Q$ and the quantum efficiency $n$ for different values of $\mathrm{M}$.

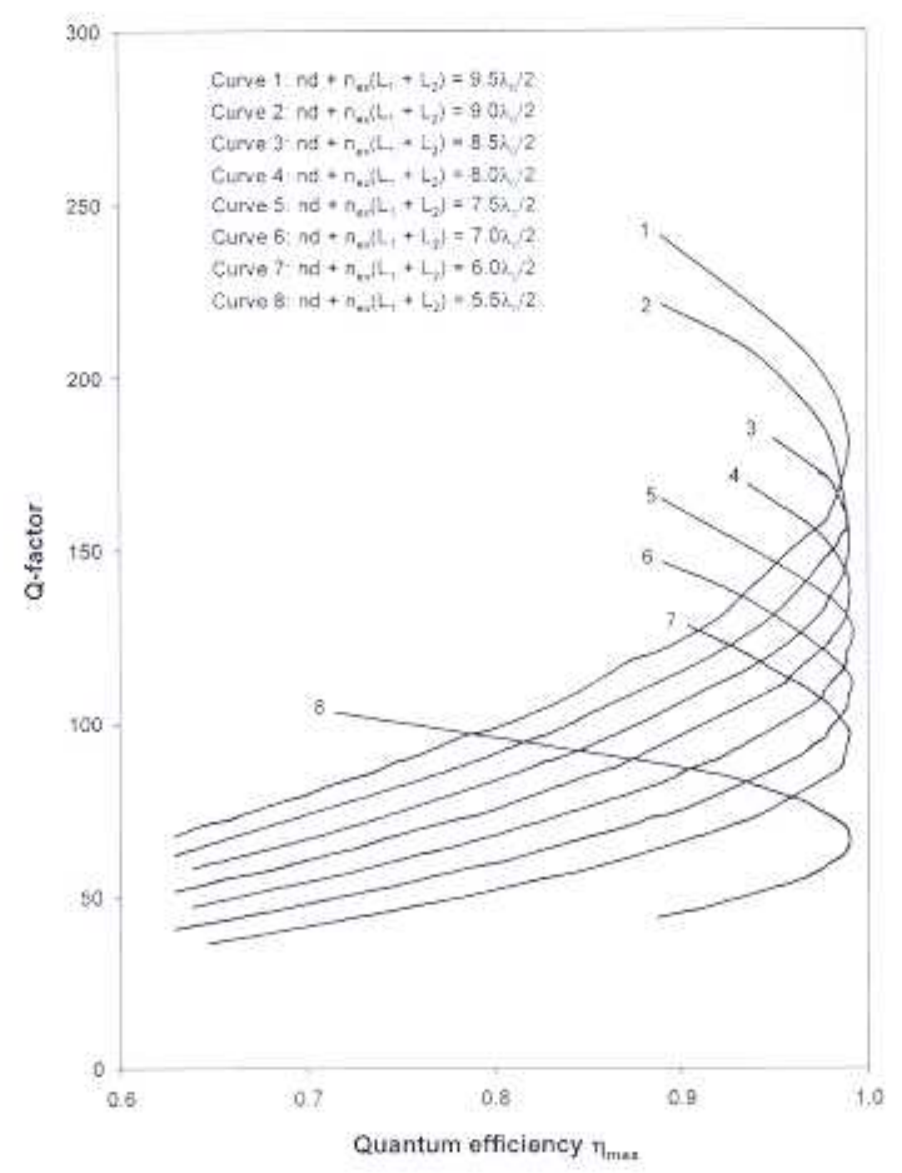

Figure 3. Design charts relating $Q$ and $\eta$ for different optical length of photodetector.

If a photodetector is to be optimized for both high Q factor and high quantum efficiency. one should select the design curve corresponding to the smallest value of $\mathrm{M}$. Once the choice of $\mathrm{Q} . \eta_{\max }$ and $\mathrm{M}$ is made, the subsequent step is to determine the geometrical parameters of the structure, namely $d, L_{1} . L_{2}$. In generaI, two possible values for $d$ can be obtained for a given $\eta_{\text {max }}$. The smallest should be chosen, as it will yield the larger frequency bandwidth. Substitution of this value of $d$ and the value of $M$ in Eq. 5 results in a linear equation with $L_{1}$ and $L_{2}$ as unknowns. An infinite number of combinations of $L_{1}$ and $L_{2}$ satisfy this equation. The choice of $\mathrm{L}_{1}=\mathrm{kL}_{2}$, (where $\mathrm{k}$ is a constant such that $0<\mathrm{K}<1$ ) does not affect the design since optical absorption in the adjacent regions is neglected.

The other important parameter in the design of RCE photodetectors is the bandwidthefficiency product (BWE). The carrier-transit time limited 3-dB bandwidth BW may be obtained using Kato's expression (Kato, 1999).

$$
B W=\frac{3.5 v}{2 \pi d}
$$

Where $v$ is the effective carrier saturation velocity and $d$ is the carrier transit distance and in this case is the absorption width.

For InGaAs, which is the absorption material used in this simulation, $v=5.3 \times 10^{4} \mathrm{~m} / \mathrm{s}$. The results of simulation are shown in Figure 4 for $L_{1}=L_{2}=1.617 \mu m$. It shows that as $d$ is 
varied, quantum efficiency increases, peaks at $d=0.1 \mu \mathrm{m}$ and then decreases. Thus, there is an optimum value for $d$, which achieves maximum quantum efficiency. The BW and BWE on the other hand, decrease with increasing $d$. Thus, for $d=0.1 \mu \mathrm{m}$, which corresponds to maximum quantum efficiency of 0.99 the BWE is $290 \mathrm{GHz}$. It is worth pointing out here that the simulated values for BWE merely serve as upper limits for the bandwidth-efficiency product. In practice, other parasitic factors, such as the leakage $\mathrm{RC}$, will lower the achievable values.

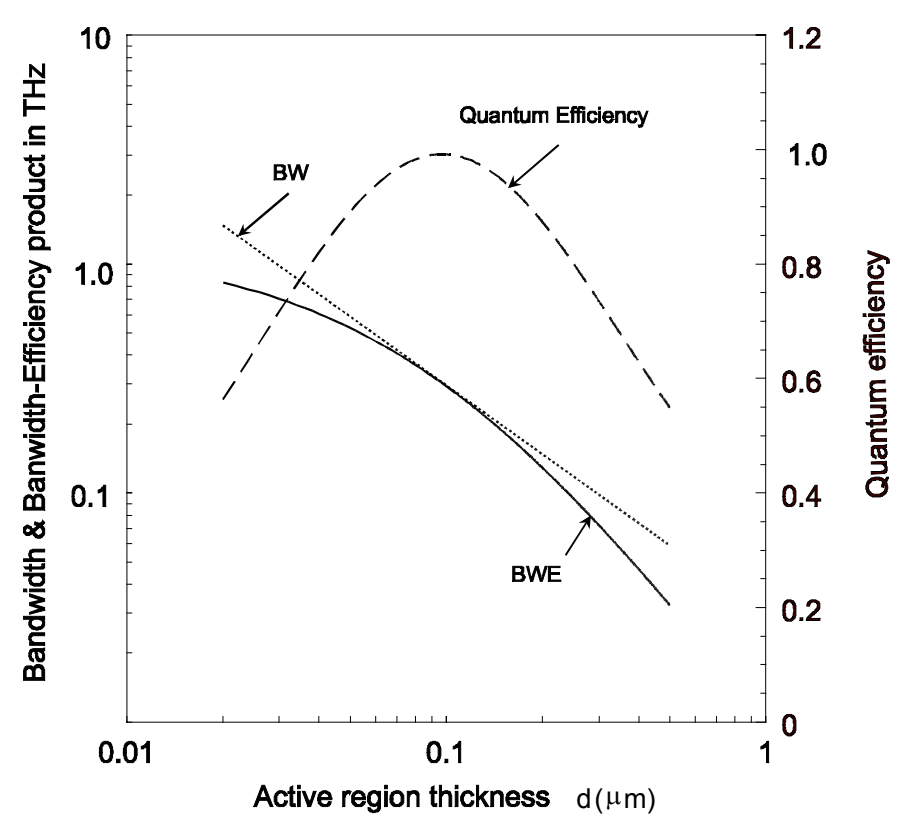

Figure 4. Variation of efficiency $\eta$ and bandwidth-efficiency product $B W E$ with active region width $d$.

\section{Conclusion}

The quantum efficiency and bandwidth-efficiency product of resonant-cavity enhanced photodetectors (RCE) has been formulated in closed analytical forms that incorporate device structural parameters. A search and optimization-based procedure has been developed with the quantum efficiency, quality factor and frequency bandwidth as input design parameters. Design charts relating the quality factor $Q$ to the maximum achievable quantum efficiency $\eta_{\max }$ for a given structure dimensions have also been generated.

\section{References}

BOTTCHER, E.H., KUHL, D., HIERONYMI, F., DROGE, E., WOLF, T. and BIMBERG, D. 1992. Ultrafast semi-insulating InP:Fe-InGaAs:Fe-InP:Fe MSM photodetectors: Modeling and Performance, IEEE J. Quantum Electron., 28: 2343-2357.

DENTAN, M. and CREMOUX, D.DE. 1990. Numerical solution of the nonlinear response of a $p$ $i$-n photodiode under high illumination, J. Lightwave Technol., 8: 1137-1144.

HUMPHREYS, D.A., KING, R.J., JENKINS, D., and MOSELEY, A.J. 1985. Measurement of absorption coefficients of $\mathrm{Ga}_{0.47} \mathrm{In}_{0.53}$ As over the wavelength range 1.0-1.7 $\mu \mathrm{m}$, Electron. Lett., 21: 1187-1189.

JERVASE, J.A. and BOURDOUCEN, H. 2000. Design of resonant-cavity-enhanced photodetectors using genetic algorithms, IEEE J. Quantum Electron., 36: 325-332.

JERVASE, J.A. and ZEBDA, Y. 1998. Characteristic analysis of resonant cavity enhanced (RCE) photodetectors, IEEE J. Quantum Electron., 34: 1129-1134. 


\section{DESIGN OF ULTR FAST RCE PHOTODETECTORS}

KATO, K. 1999. Ultrawide-Band/High-Frequency Photodetectors", IEEE Trans. Microwave Theory Tech., 47: 1265-1281.

KISHINO, K., SELIM, M.U., CHYI, REED, J.I., ARSENAULT, L., and MORKOC, H. 1991. Resonant cavity-enhanced (RCE) photodetectors", IEEE J. Quantum Electron., 27: 20252034.

KOVAC, J., UHEREK, F., SATKA, A., JAKABOVIC, J., SRNANEK, R., RHEINLANDER, B., GOTTSCHALCH, V., HASENOHRL, S., NOVAK, J., BARNA, P., BARNA, A. and WOOD, J. 1996. InAlGaAs-InGaAs-InP RCE pin photodiode for $1300 \mathrm{~nm}$ wavelength region, IPRM'96 Proc., Apr. 21-25, Germany, pp 219-222.

MURTAZA, S.S., NIE, H., CAMPELL, J.C., BEAN, J.C. and PETICOLAS, L.J. 1996. Shortwavelength, high-speed Si-based resonant-cavity photodetectors, IEEE Photon. Technol. Lett., 8: $927-929$.

MURTAZA, S.S., TAN, I.-H., BOWERS, J.E., HU, E.L., ANSELM, K.A., ISLAM, M.R., CHELAKARA, R.V., DUPUIS, R.D., STREETMAN, B.G., and CAMPBELL, J.C. 1996, High-finesse resonant-cavity photodetectors with an adjustable resonance frequency, IEEE $J$. Lightwave Technol., 14: 1081-1089.

ONAT, B.M., GOKKAVAS, OZBAY, M., E., ATA, E.P., TOWE, E., and UNLU, M.S. 1998. 100GHz resonant cavity enhanced Schottky photodiodes", IEEE Photon. Technol. Lett., 10: 707709.

OZBAY, E., SAIFUL ISLAM, M., ONAT, B., GOKKAVAS, AYTUR, M.O., TUTTLE, G., TOWE, E., HENDERSON, R.H., and UNLU, M.S. 1997. Fabrication of high-speed resonantcavity-enhanced Schottky photodiodes, IEEE Photon. Technol. Lett., 9: 672-674.

SALEM, A.F. and BRENNAN, K.F. 1995. Theoretical study of the response of InGaAs metalsemiconductor-metal photodetectors, IEEE J. Quantum Electron., 31: 944-953.

TAN, I-H., HU, E.L., BOWERS, J.E., and MILLER, B.I. 1995. Modeling and performance of wafer-fused resonant-cavity enhanced photodetectors, IEEE J. Quantum Electron., 31: 18631875.

TUNG, H.-H. and LEE, C.-P. 1997. Design of a resonant-cavity-enhanced photodetector for highspeed applications, IEEE J. Quantum Electron, 33: 753-760.

UNLU, M.S. and STRITE, S. 1995. Resonant cavity enhanced photonic devices, J. Appl. Phys., 78: 607-639.

UNLU, M.S., GOKKAVAS, M., ONAT, B.M., ATA, E., OZBAY, E., MIRIN, R.P., KNOPP, K.J., BERTNESS, K.A., and CHRISTENSEN, D.H. 1998. High bandwidth-efficiency resonant cavity enhanced Schottky photodiodes for $800-850 \mathrm{~nm}$ wavelength operation, J. Appl. Lett., 72: 2727-2729.

UNLU, M.S., KISHINO, K., LIAW, H.J., and MORKOC, H. 1992. A theoretical study of cavityenhanced photodetectors with Ge and Si active regions, J. Appl. Phys., 71: 4049-4058.

Received 23 June 2001

Accepted 6 November 2001 